\title{
Author Correction: Ferroelectric ternary content-addressable memory for one-shot learning
}

Kai Ni D, Xunzhao Yin, Ann Franchesca Laguna, Siddharth Joshi (D), Stefan Dünkel, Martin Trentzsch, Johannes Müller, Sven Beyer, Michael Niemier, Xiaobo Sharon Hu and Suman Datta

Correction to: Nature Electronics https://doi.org/10.1038/s41928-019-0321-3, published online 18 November 2019.

In the version of this Article originally published, the surname of the author Johannes Müller was incorrectly given as 'Müeller'. It has now been amended in all versions of the Article.

Published online: 3 February 2020

https://doi.org/10.1038/s41928-020-0374-3

(c) The Author(s), under exclusive licence to Springer Nature Limited 2020 